\title{
Omnidirectional Cylindrical Dielectric Resonator Antenna With Dual Polarization
}

\author{
Longfang Zou, Student Member, IEEE, Derek Abbott, Fellow, IEEE, and Christophe Fumeaux, Senior Member, IEEE
}

\begin{abstract}
This letter proposes an annular cylindrical dielectric resonator antenna (DRA) with two-port diversity realizing simultaneously omnidirectional horizontally and vertically polarized radiation patterns with low cross coupling. The horizontally and vertically polarized radiation patterns are achieved by exciting the orthogonal $\mathrm{TE}_{01 \delta}$ and $\mathrm{TM}_{01 \delta}$ modes in a single cylindrical dielectric resonator (DR). Due to the high $Q$-factor of the $\mathrm{TE}_{01 \delta}$ mode, an air gap and multiple feeding lines are introduced to increase the impedance bandwidth. The proposed antenna provides an impedance bandwidth of $19.1 \%$ in the vertically polarized mode and an overlapping bandwidth of $7.4 \%$ in the horizontally polarized mode, with the overlapping band ranging from 3.78 to 4.07 GHz. Antenna diversity and multiple-input-multiple-output (MIMO) performance are examined in terms of envelope correlation coefficient and mean effective gain.
\end{abstract}

Index Terms-Dielectric resonator antenna (DRA), multifunction, polarization diversity.

\section{INTRODUCTION}

$\mathbf{S}$ INCE a dielectric resonator antenna (DRA) was first proposed by Long in the 1980s [1], extensive research has been carried out on analyzing DRA shapes, resonant modes, radiation characteristics, and excitation schemes. The results of these investigations have highlighted attractive features of DRAs such as compact size, high radiation efficiency, and versatility in shape and feeding mechanism [2], [3]. In particular, the 3-D structure of the DRA offers an additional freedom in exciting various modes in one antenna volume. Each mode can be employed for a different application, which makes the DRA a candidate for multifunction, diversity, and multiple-input-multiple-output (MIMO) communication systems.

Several multimode usages of a single dielectric resonator (DR) have been demonstrated recently. Jazi and Denidni proposed an ultrawideband DRA by exciting the fundamental and higher-order modes [4]. Radiator and filter functions were demonstrated in a cylindrical DR by using the $\mathrm{HEM}_{11 \delta}$ and $\mathrm{TE}_{01 \delta}$ modes [5], respectively. A dual-band antenna with two different radiation characteristics in two separate bands was achieved by exciting the $\mathrm{HEM}_{11 \delta}$ and $\mathrm{TM}_{01 \delta}$ modes in a cylindrical DR in [6]. A filter function, operating in the $\mathrm{TE}_{01 \delta}$ mode, was added to the previous design to achieve a dual-band

Manuscript received April 18, 2012; accepted May 05, 2012. Date of publication May 14, 2012; date of current version May 22, 2012. The work of C. Fumeaux was supported by the Australian Research Council (ARC) Future Fellowship funding scheme under Project no. FT100100585.

The authors are with the School of Electrical and Electronic Engineering, The University of Adelaide, Adelaide, SA 5005, Australia (e-mail: 1fzou@eleceng.adelaide.edu.au; dabbott@eleceng.adelaide.edu.au; cfumeaux@eleceng.adelaide.edu.au).

Color versions of one or more of the figures in this letter are available online at $\mathrm{http}: / /$ ieeexplore.ieee.org.

Digital Object Identifier 10.1109/LAWP.2012.2199277

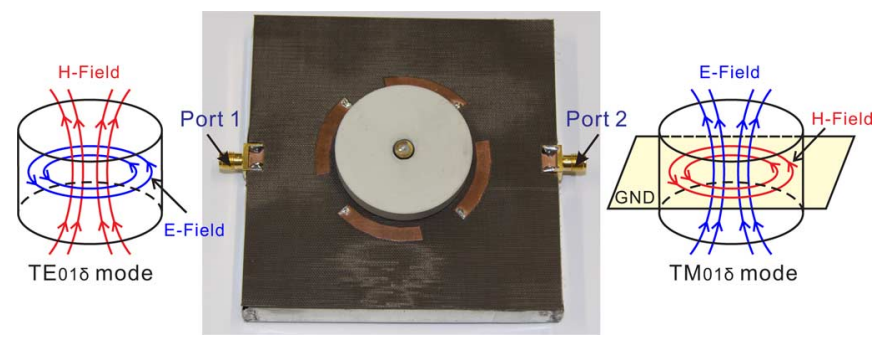

Fig. 1. Prototype of the proposed dual polarized omnidirectional DRA with schematic field distributions for the two orthogonal modes excited.

radiator and filter applications in a DR [7]. A cross-shaped DRA was designed for both multifunction and diversity applications by employing the quasi- $\mathrm{TM}_{111}$ and $\mathrm{TE}_{111}$ mode operating in an overlapping frequency band in [8]. A MIMO DRA was illustrated in a split-cylindrical DR by exciting the $\mathrm{TE}_{011+\delta}$ and $\mathrm{HE}_{01 \delta}$ modes in [9].

Due to the high quality $(Q)$ factor of the $\mathrm{TE}_{01 \delta}$ mode in a cylindrical DR, it is traditionally used in filter design [5], [7]. An attempt to obtain a horizontally polarized pattern by exciting this $\mathrm{TE}_{01 \delta}$ mode is found in [10]. However, only a $2 \%$ impedance bandwidth is achieved in simulation, and the impedance is mismatched in the prototype. By optimizing the feeding network and an air gap between the resonator and the substrate, an impedance bandwidth of $5.6 \%$ was demonstrated in both simulation and measurement in [11]. To extend this concept, we propose a dual-mode DRA with a magnetic-monopole-like and electric-monopole-like radiation patterns obtained simultaneously by exciting the $\mathrm{TE}_{01 \delta}$ and $\mathrm{TM}_{01 \delta}$ modes in a single annular cylindrical DR. Two groups of four radially arranged microstrip feeding lines with two different lengths are utilized to increase the bandwidth of the $\mathrm{TE}_{01 \delta}$ mode and suppress the influence of higher-order modes. The overlapping operating band for the two functions makes the proposed antenna suitable for being used in diversity or MIMO communication systems when omnidirectional characteristics are required.

\section{Dual Polarized DRA Design}

The prototype of the proposed dual-polarized antenna and schematic $\mathrm{TE}_{01 \delta}$ - and $\mathrm{TM}_{01 \delta}$ - mode field distribution are shown in Fig. 1. An annular cylindrical DR is mounted on a Rogers Ultralam substrate with thickness of $1.524 \mathrm{~mm}$ and relative permittivity of 2.5. The two functions of the antennas are fed through two SMA connector ports located on the left and right sides of substrate. The feeding network is located on the backside of the ground plane and shielded in a metallic cavity. A schematic of the proposed dual-polarized antenna is illustrated in Fig. 2, with all dimensions listed in Table I. The design procedure is explained in the following. 

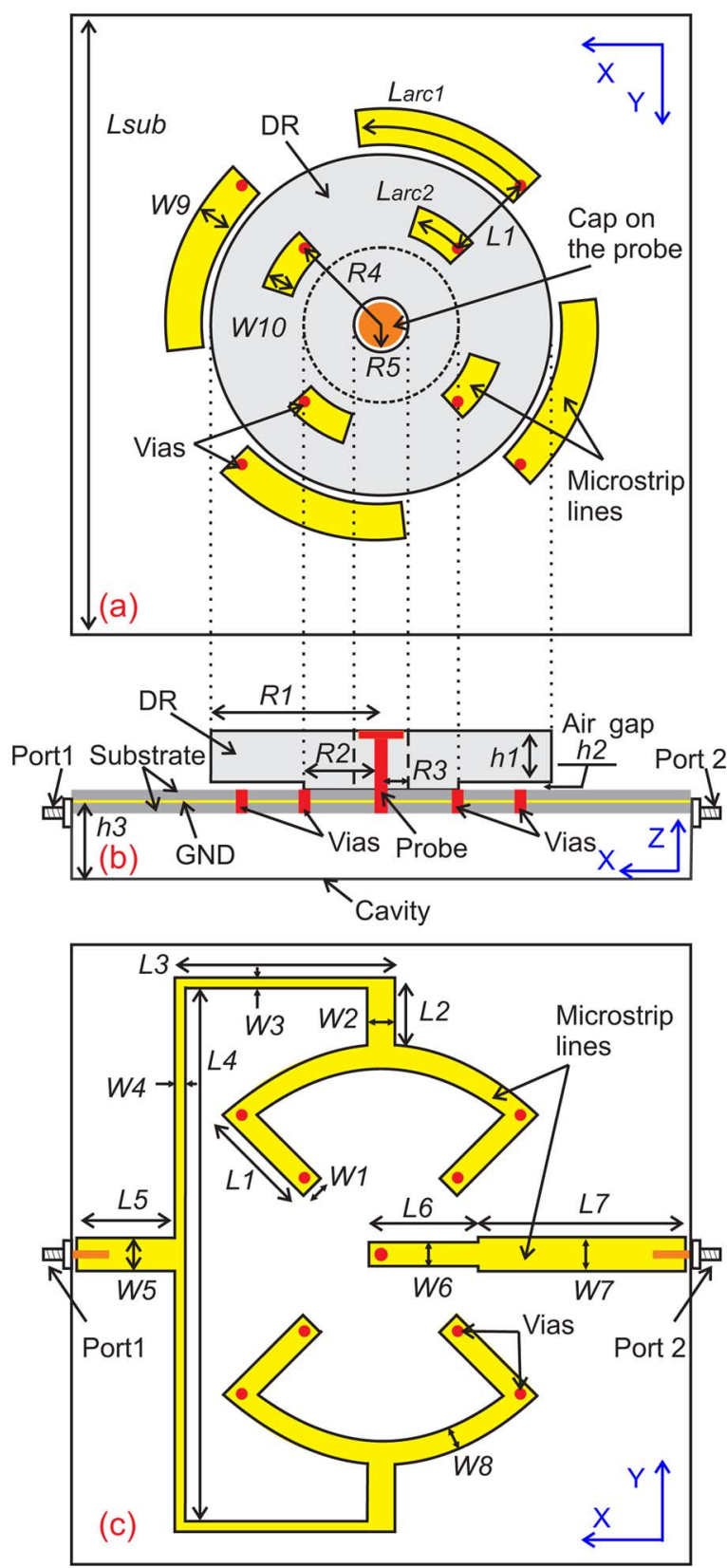

Fig. 2. Geometry of the proposed dual-polarized DRA. (a) Top view. (b) Side view. (c) Bottom view (without cavity).

The horizontally and vertically polarized radiation patterns are realized by exciting the orthogonal $\mathrm{TE}_{01 \delta}$ and $\mathrm{TM}_{01 \delta}$ modes from Ports 1 and 2, respectively. The design is carried out in three steps. First, the DR geometry is calculated. Second, the feeding network for the $\mathrm{TE}_{01 \delta}$ mode is realized. Third, the design is extended to include the feeding network for the $\mathrm{TM}_{01 \delta}$ mode. The DR and feeding networks are simulated and optimized with Ansoft HFSS.

\section{A. DR Design}

A cylindrical DR is first designed to excite the horizontally polarized $\mathrm{TE}_{01 \delta}$ mode resonant at $3.9 \mathrm{GHz}$. The initial size of the DR is calculated based on closed-form formulas given in [3]. An air gap is introduced between the DR and the substrate to increase distance between DR and ground and hence widen the impedance bandwidth of this $\mathrm{TE}_{01 \delta}$ mode [11]. The DR is cut

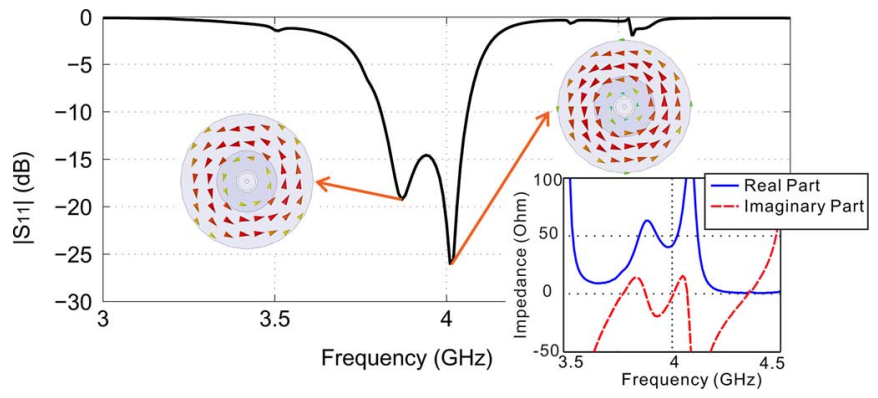

Fig. 3. Simulated reflection coefficient of Port 1. Inset: $\mathrm{TE}_{01 \delta}$ mode electric field distribution inside the dielectric resonator at 3.87 and $4.02 \mathrm{GHz}$. Inset: Real and imaginary part of the input impedance.

TABLE I

ANTENNA PARAMETERS

\begin{tabular}{|c|c|c|c|c|c|}
\hline Symbol & $\begin{array}{c}\text { Value } \\
(\mathrm{mm})\end{array}$ & Symbol & $\begin{array}{c}\text { Value } \\
(\mathrm{mm})\end{array}$ & Symbol & $\begin{array}{c}\text { Value } \\
(\mathrm{mm})\end{array}$ \\
\hline$L_{1}$ & 12 & $L_{2}$ & 13 & $L_{3}$ & 35 \\
\hline$L_{4}$ & 78 & $L_{5}$ & 13.5 & $L_{6}$ & 14 \\
\hline$L_{7}$ & 36.5 & $W_{1}$ & 3 & $W_{2}$ & 3.5 \\
\hline$W_{3}$ & 1.22 & $W_{4}$ & 1.22 & $W_{5}$ & 4.35 \\
\hline$W_{6}$ & 3 & $W_{7}$ & 4.35 & $W_{8}$ & 3 \\
\hline$W_{9}$ & 4.5 & $W_{10}$ & 4 & $L_{\text {sub }}$ & 100 \\
\hline$h_{1}$ & 6.62 & $h_{2}$ & 1 & $h_{3}$ & 10 \\
\hline$R_{1}$ & 22 & $R_{2}$ & 10 & $R_{3}$ & 3.5 \\
\hline$R_{4}$ & 14 & $R_{5}$ & 2.9 & $L_{\text {arc1 }}$ & 22.7 \\
\hline$L_{\mathrm{arc} 2}$ & 6.4 & & & & \\
\hline
\end{tabular}

from a dielectric slab with thickness of $7.62 \mathrm{~mm}$ and relative permittivity of 10 . For simplicity of manufacturing, the DR is kept at the slab height of $7.62 \mathrm{~mm}$. The size of DR can be scaled up or down for operation at other frequencies.

\section{B. Excitation of $T E_{01 \delta}$ Mode}

In [11], the impedance bandwidth of the $\mathrm{TE}_{01 \delta}$ mode was increased to $5.6 \%$ by using two long and wide curved microstrip feeding lines. However, this bandwidth improvement was obtained at the cost of the increase in cross polarization due to the detrimental effect of the coupling with the higher mode $\mathrm{HEM}_{21 \delta}$. In the presently proposed design, two groups of four arc-shaped microstrip lines of different length and width are utilized to excite the DR, as shown in Fig. 2. By using this novel feeding method, the simulated impedance bandwidth, shown in Fig. 3, is further increased to $7.4 \%$ due to the combination of overlapping resonances excited by these two group feeding lines. The real and imaginary parts of the input impedance shown in the inset demonstrates the branching into two resonant frequencies. The electric field distribution for the first resonant mode at $3.87 \mathrm{GHz}$ and the second one at $4.02 \mathrm{GHz}$ are illustrated in Fig. 3. These field plots confirm that both resonant frequencies are related to the excitation of the $\mathrm{TE}_{01 \delta}$ mode in the DR. In addition, increasing the number of feeding groups to four suppresses the radially asymmetric $\mathrm{HEM}_{21 \delta}$ mode.

In the feeding network design process, a group of $50-\Omega$ curved half-wavelength microstrip lines $\left(L_{\mathrm{arc1}}\right)$ are first designed to excite a single resonant frequency. The feeding lines 


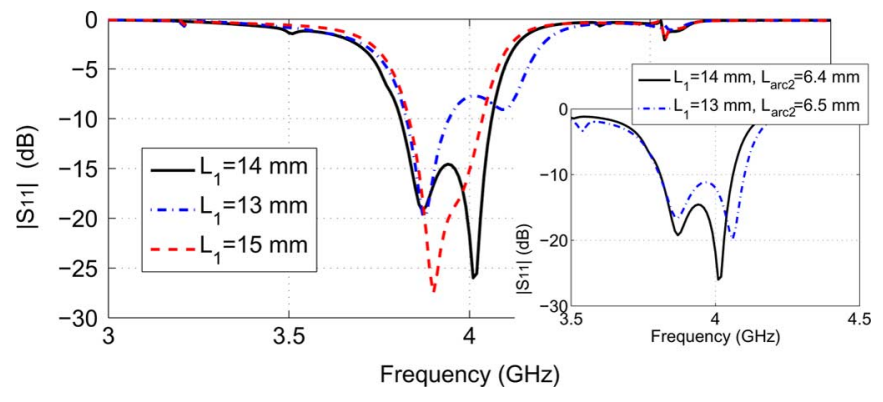

Fig. 4. Simulated reflection coefficient of Port 1 with different length of $L_{1}$. Inset: Further bandwidth increase by optimizing length of $L_{\mathrm{arc} 2}$ and $L_{1}$.

are initially placed at the outer periphery of the DR to achieve a good excitation efficiency and leave space for the inner feeding lines. The width of the feeding lines is slightly adjusted to match the feeding network. The location of the feeding lines is further moved out from the DR to reduce the impact on other feeding parts while keeping excitation efficiency within acceptable level.

The inner feeding lines are added to create a fork-like feeding structure and excite the higher resonant frequency. The width of the feeding lines is set to $4 \mathrm{~mm}$ to match the existing feeding network. The higher resonant frequency is related to the added length of the shorter curved lines $L_{\text {arc2 }}$ and radial microstrip line $L_{1}$, as demonstrated in Fig. 4, where the first resonant frequency remains unchanged and the higher resonant frequency moves to higher or lower frequency with the decrease or increase of the length of the radial microstrip line $\left(L_{1}\right)$, respectively. Furthermore, the inset of Fig. 4 illustrates that the impedance bandwidth can be further increased to $8.1 \%$ by moving the second resonant frequency toward higher frequency through the optimization of the length $L_{1}$ and $L_{\mathrm{arc} 2}$. However the reflection coefficient of the middle frequency band becomes too close to $-10 \mathrm{~dB}$. Considering the manufacturing accuracy, the conservatively chosen length $L_{1}=14 \mathrm{~mm}$ was used to realize a prototype.

In the manufacture, two substrates are cut from a Rogers U1tralam substrate with backside metallization. The curved microstrip feeding lines are printed on the top of one substrate, and the feeding networks are printed on the other. Then, the backside metal of these two substrates are attached together to form the ground plane. In order to reduce the impact from the feeding network, it is physically separated from the microstrip feeding lines by the ground plane and sealed in a metal cavity. Vias with a diameter of $1 \mathrm{~mm}$ are employed to connect the feeding network and the arc-shaped microstrip feeding lines through holes in the ground plane and substrates.

To connect the multiple arc-shaped feeding structure to Port 1, several T-junctions are printed on the bottom of the substrate. The first T-junction [located on left side in Fig. 2(c)] is utilized to connect the 50- $\Omega$ transmission line from the SMA connector (Port 1) to two 100- $\Omega$ transmission lines. Furthermore, as seen on the top and bottom of Fig. 2(c), the 100- $\Omega$ line branches out to two $31-\Omega$ arcs, with matching realized through quarter-wave transformers (with length $L_{2}$ and width $W_{2}$ ).

\section{Excitation of the $T M_{01 \delta}$ Mode}

The center of cylindrical DR is removed to provide room for a central probe feed. The introduction of the annular geometry for

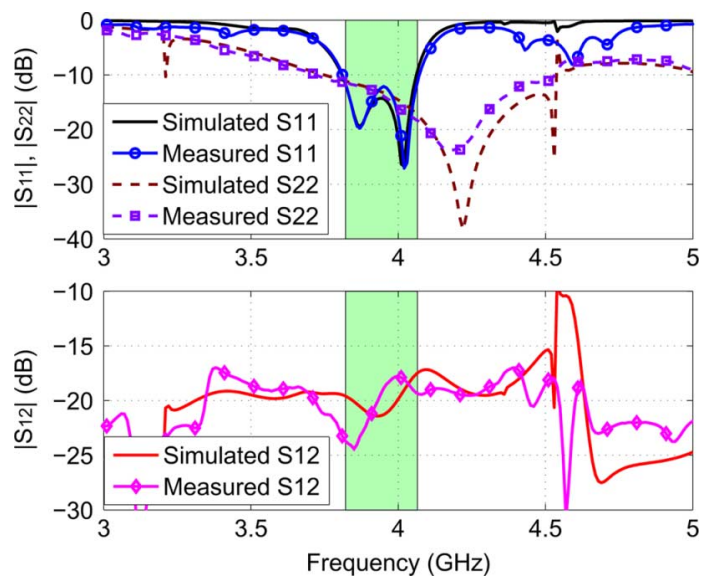

Fig. 5. Simulated and measured magnitude of (a) the antenna reflection coefficient for both ports and (b) interport coupling coefficient.

the $\mathrm{TM}_{01 \delta}$-mode probe excitation does not influence markedly the $\mathrm{TE}_{01 \delta}$ mode, which has a zero E-field in the center. For diversity and MIMO operation of the antenna, the impedance band of the two ports should overlap as much as possible to maximize the operation bandwidth. However, the resonant frequency of the pure $\mathrm{TM}_{01 \delta}$ mode is higher than that of the $\mathrm{TE}_{01 \delta}$ mode. Therefore, in this third design step, the hybrid monopole DRA concept, described in [12], is utilized to increase the bandwidth of Port 2. A metal cap is added on top of the electric monopole to shorten it and hence lower the overall antenna profile [13]. The inner radius of the annular cylindrical DR and cap are adjusted to widen the bandwidth response of Port 2 and maximize its overlap with the bandwidth of Port 1 . The monopole is connected to a $50-\Omega$ microstrip line by a quarter-wavelength transmission line matching section, located at the bottom of the substrate, as shown on the right side of Fig. 2(c).

\section{ANTENNA PERformance}

The reflection coefficients of Ports 1 and 2 are shown in Fig. 5(a), exhibiting a good agreement between simulation and measurement. The Port 1 excites the $\mathrm{TE}_{01 \delta}$ mode and presents an impedance bandwidth $\left(\left|S_{11}\right|<-10 \mathrm{~dB}\right)$ of $7.4 \%$, whereas the Port 2 excites the $\mathrm{TM}_{01 \delta}$ mode with a $19.1 \%$ impedance bandwidth. The shaded area shows the usable overlapping band, which corresponds to the Port-1 impedance working frequency band, from 3.78 to $4.07 \mathrm{GHz}$. As shown in Fig. 5(b), the mutual coupling between these two ports is well below -15 $\mathrm{dB}$ in the overlapping band.

Omnidirectional radiation patterns are measured in the overlapping band of the two ports. The radiation patterns at the center of the band $(3.93 \mathrm{GHz})$ are shown as an illustrative example in the following. Fig. 6 depicts simulated and measured radiation patterns of Port 1 in the $x z$ - and $y z$-planes. An omnidirectional horizontally polarized conical pattern is obtained with measured copolarization levels at least $15 \mathrm{~dB}$ higher than the cross-polarization levels in both planes. Symmetric patterns and low cross polarization are obtained because of the symmetry of the eight-arc feeding network. For Port 2, vertically polarized electric-monopole-like radiation patterns are achieved as shown in Fig. 7 at $3.93 \mathrm{GHz}$. The patterns shown in the $x z$ and $y z$-planes have cross-polarization levels below $-20 \mathrm{~dB}$. In 


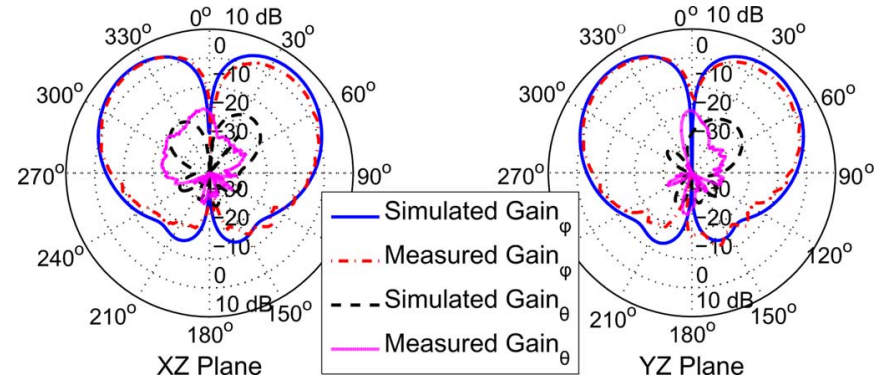

Fig. 6. Simulated and measured radiation pattern of Port 1 at $3.93 \mathrm{GHz}$.

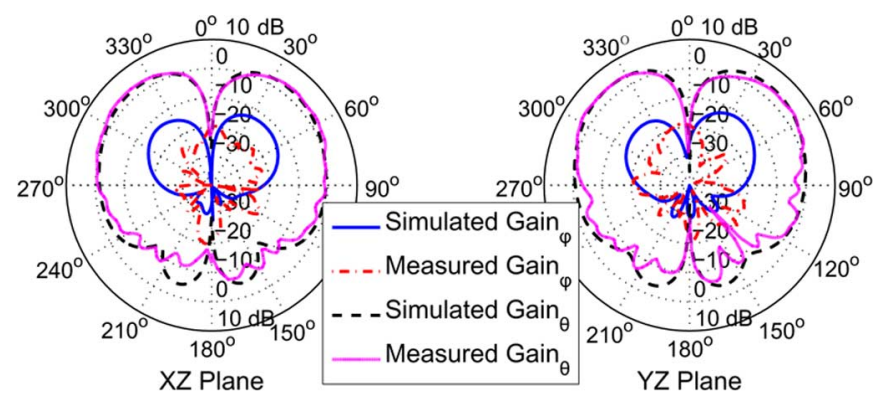

Fig. 7. Simulated and measured radiation pattern of Port 2 at $3.93 \mathrm{GHz}$.

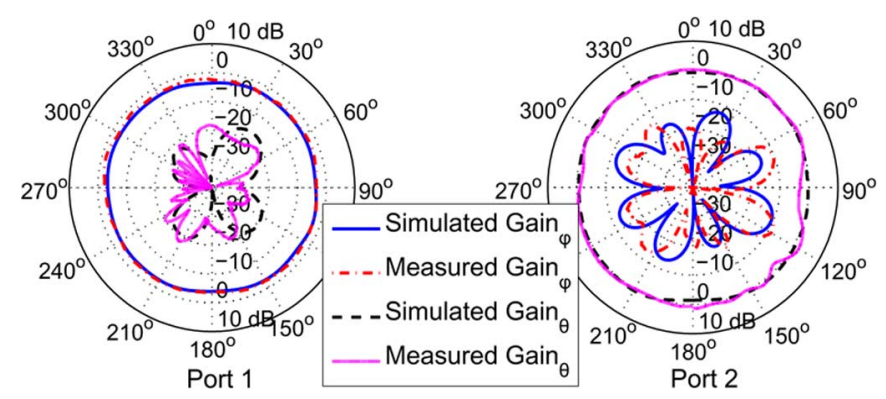

Fig. 8. Radiation patterns of Ports 1 and 2 at $3.93 \mathrm{GHz}$ in $x y$-plane.

Fig. 8, the patterns of Ports 1 and 2 in the $x y$-plane shows omnidirectional characteristics. In the overlapping band, the average gain at $\theta=45^{\circ}$ is at the range of $5.1-5.3 \mathrm{~dB}$ for the horizontally polarized pattern, and between $1.7-2.1 \mathrm{~dB}$ for the vertically polarized one.

The overlapping working bandwidth for both modes suggests the application of the proposed antenna as a diversity or MIMO antenna. To evaluate the diversity characteristics and MIMO performance of the proposed device, the envelope correlation (EC) [14] and mean effective gain (MEG) [15] are calculated using $S$-parameters for both simulation and measurement results. The calculated results in Fig. 9 satisfy the criteria of low correlation $(\mathrm{EC}<0.5)$ and comparable average received power $\left(\mathrm{MEG}_{1}\right.$ and $\mathrm{MEG}_{2}>-10 \mathrm{~dB}$, $\left|\mathrm{MEG}_{1}-\mathrm{MEG}_{2}\right|<3 \mathrm{~dB}$ ). Thus, a good diversity performance of the proposed antenna can be expected.

\section{CONCLUSION}

This letter has proposed a dual-mode annular cylindrical DRA able to operate simultaneously with horizontally and vertically polarized omnidirectional radiation characteristics. The horizontally polarized radiation patterns is achieved by exciting the $\mathrm{TE}_{01 \delta}$ mode of the $\mathrm{DR}$, with an impedance bandwidth increased to $7.4 \%$ by using an air gap and double microstrip feeding arcs. In contrast, the omnidirectional $\mathrm{TM}_{01 \delta}$ radiating

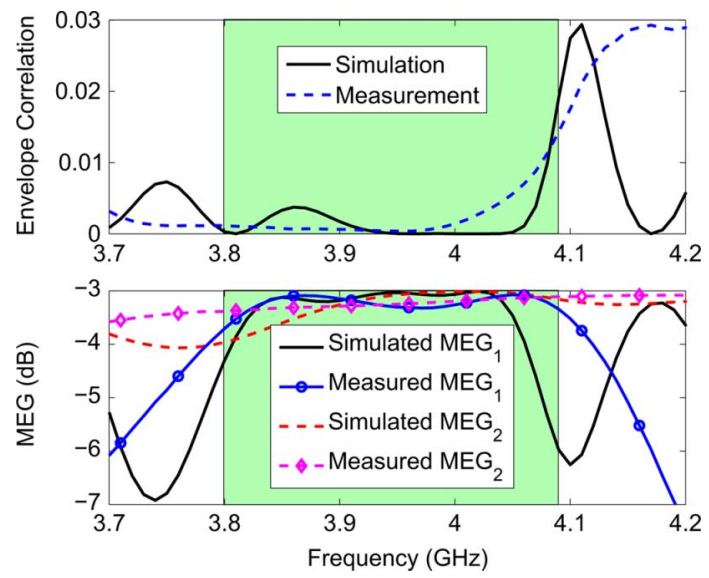

Fig. 9. Simulated and measured envelope correlation coefficients and mean effective gain.

mode yields a vertically polarized monopole-type radiation pattern. By exploiting the hybrid monopole-DRA structure, the bandwidth of the $\mathrm{TM}_{01 \delta}$ mode is increased to $19.1 \%$ and overlaps with the bandwidth of the $\mathrm{TE}_{01 \delta}$ mode. The calculated EC and MEG indicates a good diversity performance across the common band from 3.78 to $4.07 \mathrm{GHz}$.

\section{REFERENCES}

[1] S. Long, M. McAllister, and L. Shen, "The resonant cylindrical dielectric cavity antenna," IEEE Trans. Antennas Propag., vol. AP-31, no. 3, pp. 406-412, May 1983.

[2] A. Petosa and A. Ittipiboon, "Dielectric resonator antennas: A historical review and the current state of the art," IEEE Antennas Propag. Mag., vol. 52, no. 5, pp. 91-116, Oct. 2010.

[3] K. M. Luk and K. W. Leung, Dielectric Resonator Antennas. Baldock, England: Res. Studies Press, 2003.

[4] M. N. Jazi and T. A. Denidni, "Design and implementation of an ultrawideband hybrid skirt monopole dielectric resonator antenna," IEEE Antennas Wireless Propag. Lett., vol. 7, pp. 493-496, 2008.

[5] E. H. Lim and K. W. Leung, "Use of the dielectric resonator antenna as a filter element," IEEE Trans. Antennas Propag., vol. 56, no. 1, pp. 5-10, Jan. 2008.

[6] L. K. Hady, A. A. Kishk, and D. Kajfez, "Dual-band compact DRA with circular and monopole-like linear polarizations as a concept for GPS and WLAN applications," IEEE Trans. Antennas Propag, vol. 57, no. 9, pp. 2591-2598, Sep. 2009.

[7] L. K. Hady, D. Kajfez, and A. A. Kishk, "Triple mode use of a single dielectric resonator," IEEE Trans. Antennas Propag., vol. 57, no. 5, pp. 1328-1335, May 2009.

[8] L. Zou and C. Fumeaux, "A cross-shaped dielectric resonator antenna for multifunction and polarization diversity applications," IEEE Antennas Wireless Propag. Lett., vol. 10, pp. 742-745, 2011.

[9] J.-B. Yan and J. T. Bernhard, "Design of a MIMO dielectric resonator antenna for LTE femtocell base stations," IEEE Trans. Antennas Propag., vol. 60, no. 2, pp. 438-444, Feb. 2012.

[10] U. M. Khan and M. Milutinovic, "Dual polarized dielectric resonator antennas," Master's thesis, Chalmers Univ. Technol., Goteborg, Sweden, 2010.

[11] L. Zou and C. Fumeaux, "Horizontally polarized omnidirectional dielectric resonator antenna," in Proc. APMC, Dec. 2011, pp. 849-852.

[12] M. Lapierre, Y. Antar, A. Ittipiboon, and A. Petosa, "Ultra wideband monopole/dielectric resonator antenna," IEEE Microw. Wireless Compon. Lett., vol. 15, no. 1, pp. 7-9, Jan. 2005.

[13] G. Almpanis, C. Fumeaux, J. Fröhlich, and R. Vahldieck, "A truncated conical dielectric resonator antenna for body-area network applications," IEEE Antennas Wireless Propag. Lett., vol. 8, pp. 279-282, 2009.

[14] S. Blanch, J. Romeu, and I. Corbella, "Exact representation of antenna system diversity performance from input parameter description," Electron. Lett., vol. 39, no. 9, pp. 705-707, May 2003.

[15] T. Taga, "Analysis for mean effective gain of mobile antennas in land mobile radio environments," IEEE Trans. Veh. Technol., vol. 39, no. 2, pp. 117-131, May 1990. 